\title{
Proper Reparametrization of Rational Ruled Surface ${ }^{1}$
}

\author{
Jia Li, Liyong Shen, and Xiao-Shan Gao \\ Key Laboratory of Mathematics Mechanization \\ Institute of Systems Science, AMSS, Academia Sinica \\ Beijing 100080, China \\ (xgao,lijia)@mmrc.iss.ac.cn, shenly@amss.ac.cn
}

\begin{abstract}
In this paper, we present a proper reparametrization algorithm for rational ruled surfaces. That is, for an improper rational parametrization of a ruled surface, we construct a proper rational parametrization for the same surface.
\end{abstract}

Keywords. Proper reparametrization, ruled surface, algebraic curve.

\section{Introduction}

Rational parametric curves and surfaces are one of the main tools in geometric modeling. A key property of a set of rational parametrization is whether the rational parametrization is proper, that is, whether the values of the parameters and the image points are in a one to one correspondence. For instance

$$
x=\frac{2 t}{t^{2}+1}, y=\frac{t^{2}-1}{t^{2}+1}
$$

are a proper parametrization for the unit circle $x^{2}+y^{2}=1$, while

$$
x=\frac{2 t^{2}}{t^{4}+1}, y=\frac{t^{4}-1}{t^{4}+1}
$$

are also a parametrization for the unit circle, but they are improper. Because for a point $(x, y)$ on the circle, there exist two corresponding parameters $t= \pm \sqrt{\frac{x}{1-y}}$. Improper parametrizations are undesirable because the parametric degree could be unnecessarily high. So a natural question is: whether we can find a proper reparametrization for a set of improper rational parametrizations?

In the case of algebraic curves, the answer is positive. Based on Lüroth's theorem [11], various proper reparametrization algorithms for rational parametrizations of algebraic curves are developed, such as the Gröbner basis method, the characteristic set method, and the GCD method $[5,6,9,10]$.

In the case of algebraic surfaces, we can determine whether a surface is proper using the $u$-resultant [3] or the Gröbner basis [5]. However, the problem of finding a proper reparametrization for an improper rational parametrization of an algebraic surface is open in the

\footnotetext{
${ }^{1)}$ This paper is partially supported by a national key basic research project of China.
} 
general case [6]. There exist several partial results. In [10], a proper reparametrization algorithm was proposed for rational parametrizations which are improper in each parameter independently, that is, the proper reparametrization can be found by replacing each parameter with a rational function in itself. In [8], a proper reparametrization algorithm was proposed for rational parametrizations which are improper in only one of the parameters. In [4], a class of inherently improper parametric supports was studied.

Rational ruled surfaces are an important class of algebraic surfaces widely used in geometric modeling. Even for this simple class of surfaces, proper reparametrization algorithms do not exist. In $[1,2]$, the $\mu$-basis was used to reparameterize rational ruled surface to obtain a new parametrization with lower degrees. But, the new parametrization is not necessarily proper.

In this paper, we give a solution to the proper reparametrization problem for ruled algebraic surfaces given by a set of rational parametrizations. Our algorithm works as follows. The improper rational parametrization is first transformed to a new one which is proper for one of the variables. Then, by considering the improper parameter only, the rational parametrization can be treated as a rational parametrization of an algebraic curve and the proper parameter is considered to be in the coefficients. Finally, we find a proper reparametrization for this curve with known methods and show that this reparametrization also provides a proper reparametrization for the ruled surface. The algorithm is implemented and experimental results show that the algorithm is quite effective.

The rest of this paper is organized into three sections. In Section 2, we introduce the notations and preliminary results. In Section 3, we present the reparametrize algorithm. In Section 4, conclusions are given.

\section{Notations and Preliminary Results}

In this section, we introduce the notations and preliminary results needed in our algorithm. Let $\mathbb{Q}[s]$ be the polynomial ring over the field of rational numbers, and $\mathbb{Q}[s]^{4}$ the set of four-dimensional row vectors whose entries belong to $\mathbb{Q}[s]$. A rational ruled surface in homogeneous form is defined as:

$$
(x, y, z, w)=\mathbf{P}(s, t)=\mathbf{P}_{\mathbf{0}}(s)+\mathbf{P}_{\mathbf{1}}(s) t=(a(s, t), b(s, t), c(s, t), d(s, t))
$$

where $\mathbf{P}_{\mathbf{i}}(s)=\left(a_{i}(s), b_{i}(s), c_{i}(s), d_{i}(s)\right) \in \mathbb{Q}[s]^{4}, i=0,1$, are called the directrices of $\mathbf{P}(s, t)$, $\operatorname{gcd}(a(s, t), b(s, t), c(s, t), d(s, t))=1$, and $d_{0}+d_{1} t \neq 0$. We assume that the rational parametrization (1) is nontrivial, that is, it defines a surface $f(x, y, z, w)=0 . \mathbf{P}(s, t)$ is nontrivial if and only if $\mathbf{P}_{\mathbf{0}}(s)$ and $\mathbf{P}_{\mathbf{1}}(s)$ are linear independent over $\mathbb{Q}[s]$. A reparametrization of (1) is another rational parametrization which defines the same surface $f(x, y, z, w)=0$.

The inverse problem for the rational parametrization (1) is to find the values of the corresponding parameters $s$ and $t$ for a given point on the surface $f(x, y, z, w)=0$. Equivalently, we need to solve $t$ and $s$ from the following equations [5]:

$$
\left\{\begin{aligned}
\left(d_{0}(s)+d_{1}(s) t\right) x= & \left(a_{0}(s)+a_{1}(s) t\right) w \\
\left(d_{0}(s)+d_{1}(s) t\right) y= & \left(b_{0}(s)+b_{1}(s) t\right) w \\
\left(d_{0}(s)+d_{1}(s) t\right) z= & \left(c_{0}(s)+c_{1}(s) t\right) w \\
& d_{0}(s)+d_{1}(s) t \neq 0
\end{aligned}\right.
$$


The parametrization (1) is called proper, if for a generic point on the surface, $t$ and $s$ have a unique solution, that is, from (2), we have the inverse map:

$$
t=r_{1}(x, y, z, w), s=r_{2}(x, y, z, w)
$$

where $r_{1}, r_{2} \in \mathbb{Q}(x, y, z, w)$.

The parametrization (1) is called proper for variable $t$, if for a generic point on the surface, $t$ has a unique solution, that is, from (2), we have the inverse map for $t$ :

$$
t=r_{1}(x, y, z, w)
$$

where $r_{1} \in \mathbb{Q}(x, y, z, w)$.

If a surface is not proper, then for a generic point on the surface, there exist a fixed number of parametric values corresponding to this point $[3,5,7]$. This fixed number is called the improper index of the parametrization (1), denoted by $I X(\mathbf{P})$. The improper index of (1) can be found by computing the $u$-resultant [3] or by computing the Gröbner basis of (2) [5].

One natural idea is to reparametrize (1) by finding a nontrivial decomposition

$$
a_{i}(s)=\bar{a}_{i}(h(s)), b_{i}(s)=\bar{b}_{i}(h(s)), c_{i}(s)=\bar{c}_{i}(h(s)), d_{i}(s)=\bar{d}_{i}(h(s)), i=0,1
$$

where $h(s) \in \mathbb{Q}(s)$. The following example shows that this is generally not possible for an improper parametrization (1).

Example 2.1 $\mathbf{P}(s, t)=(3 s+(s+1) t, 2 s+s t, s-1+t, 1)$ is a parametrization of the plane $x-y-z-w=0 . \mathbf{P}(s, t)$ is not proper. With the method in [5], we could show that the improper index is two. It is clear that the polynomials $3 s, s+1,2 s, s, s-1$ do not have a nontrivial decomposition like (3), so we cannot reparameterize $\mathbf{P}(s, t)$ with a set of new variables like $\bar{t}=t, \bar{s}=h(s)$. Similarly, we may check that $\mathbf{P}(s, t)$ is not proper for $t$ (or $s$ ). So, the methods in $[8,10]$ can not be used to this example.

Apply the results from [4] to rational parametrization (1) could give us some insights into the problem.

The support of $\mathbf{P}$ of form (1) is the set of $(m, n)$ such that $s^{m} t^{n}$ is a monomial in (1). Denote the support of $\mathbf{P}$ by $S(\mathbf{P})$. We assume that the supports of $a_{0}+a_{1} t, b_{0}+b_{1} t, c_{0}+c_{1} t$, and $d_{0}+d_{1} t$ are the same. It is clear that the supports of (1) could be arrange into the following form

$$
\left(g_{1}, 0\right),\left(g_{2}, 0\right), \ldots,\left(g_{p}, 0\right) ;\left(e_{1}, 1\right),\left(e_{2}, 1\right), \ldots,\left(e_{q}, 1\right)
$$

where $g_{1}<g_{2}<\cdots<g_{p}$ and $e_{1}<e_{2}<\cdots<e_{q}$. For a triangle $S^{\prime}$, the normalized area $N A\left(S^{\prime}\right)$ is twice the usual Euclidean area of $S^{\prime}$. We define the gcd of the degree gap of (1) to be $\operatorname{gcddg}(\mathbf{P})=\operatorname{gcd}\left\{g_{i+1}-g_{i}, e_{j+1}-e_{j}: i=1, \ldots, p-1 ; j=1, \ldots, q-1\right\}$. We have

Proposition 2.2 Let $\mathbf{P}(s, t)$ be a rational parametrization like (1) with support as (4). Then we have the following results.

(1) If $\operatorname{gcddg}(\mathbf{P})>1$, then $\mathbf{P}$ is improper with improper index $\operatorname{gcddg}(\mathbf{P}) \cdot$ I for a positive integer $I$. In this case, we can reparametrize $\mathbf{P}$ such that the improper index for the new parametrization is $I$. 
(2) If $\operatorname{gcddg}(\mathbf{P})=1$, then $\mathbf{P}$ with randomly chosen coefficients is proper with probability one. In other words, $\mathbf{P}$ is improper only in a lower dimensional sub-space of the coefficients space.

Proof: First, we consider the rational parametric ruled surface like (1) with generic coefficients. According to Theorem 1 of [4], the improper index of (1) is

$$
I X(\mathbf{P})=\operatorname{gcd}\left\{N A\left(S^{\prime}\right): S^{\prime} \subseteq S(\mathbf{P}),\left|S^{\prime}\right|=3\right\} .
$$

The triangle in the support set $S(\mathbf{P})$ can only be $\left\{\left(g_{i_{1}}, 0\right),\left(g_{i_{2}}, 0\right),\left(e_{j}, 1\right)\right\}$ or $\left\{\left(g_{i}, 0\right)\right.$, $\left.\left(e_{j_{1}}, 1\right),\left(e_{j_{2}}, 1\right)\right\}, i_{1}<i_{2}, j_{1}<j_{2}$. The corresponding normalized area is $g_{i_{2}}-g_{i_{1}}$ or $e_{j_{2}}-e_{j_{1}}$. Then

$$
\begin{aligned}
& I X(\mathbf{P})=\operatorname{gcd}\left\{N A\left(S^{\prime}\right): S^{\prime} \subseteq S(\mathbf{P}),\left|S^{\prime}\right|=3\right\} \\
= & \operatorname{gcd}\left\{g_{i_{2}}-g_{i_{1}}, e_{j_{2}}-e_{j_{1}}: 1 \leqslant i_{1}<i_{2} \leqslant p ; 1 \leqslant j_{1}<j_{2} \leqslant q\right\} \\
= & \operatorname{gcd}\left\{g_{i+1}-g_{i}, e_{j+1}-e_{j}: 1 \leqslant i \leqslant p-1 ; 1 \leqslant j \leqslant q-1\right\} \\
= & \operatorname{gcddg}(\mathbf{P}) .
\end{aligned}
$$

For a rational parametrization (1) with coefficients in $\mathbb{Q}$, its improper index is $I X(\mathbf{P}) \cdot I=$ $\operatorname{gcddg}(\mathbf{P}) \cdot I$ for a positive integer $I([4]$, Theorem 2$)$.

When $\operatorname{gcddg}(\mathbf{P})>1$, we can reparametrize $\mathbf{P}$ such that the improper index for the new parametrization is $I$. We need only to reparametrize $\mathbf{P}$ such that the gcd of degree gap for the new parametrization is 1 . If $(0,0)$ is not in the support $S(\mathbf{P})$. We use $s^{\prime}=s, t^{\prime}=t s^{e_{1}-g_{1}}$ to reparametrize $\mathbf{P}$ as $\mathbf{P}^{\prime}$. Let $g=\operatorname{gcddg}(\mathbf{P})$. Then the support for $\mathbf{P}^{\prime}$ consists of

$$
(0,0),\left(k_{1} g, 0\right), \ldots,\left(k_{p-1} g, 0\right),(0,1),\left(h_{1} g, 1\right), \ldots,\left(h_{q-1} g, 1\right)
$$

where $k_{i}$ and $h_{i}$ are positive integers. Then the support of the new parametrization $\mathbf{P}^{\prime}$ contains $(0,0)$ and $\operatorname{gcddg}\left(\mathbf{P}^{\prime}\right)=\operatorname{gcddg}(\mathbf{P})$. So we can reparametrize $\mathbf{P}$ with the new parameters $\bar{s}=s^{g}, \bar{t}=t$ and obtain the reparametrization $\overline{\mathbf{P}}$ of $\mathbf{P}$ such that $\operatorname{gcddg}(\overline{\mathbf{P}})=1$.

If $\operatorname{gcddg}(\mathbf{P})=1$, for coefficients of (1) taken from a Zariski open set in the coefficients space $\mathbb{Q}^{4|S(\mathbf{P})|}$, parametrization (1) is proper ([4], Theorem 3). Since the Zariski open set is the whole coefficients space minus a set with lower dimensions, we prove the proposition.

\section{Example 2.3}

$$
\mathbf{P}(s, t)=\left(s+s^{3}+\left(1+s^{2}\right) t, s+2 s^{3}+\left(1-s^{2}\right) t, s^{3}+t, s+t\right) .
$$

The support of $\mathbf{P}$ is $S(\mathbf{P})=\{(1,0),(3,0),(0,1),(2,1)\}$, and $\operatorname{gcddg}(\mathbf{P})=\operatorname{gcd}\{3-1,2-0\}=2$. $S$ is an improper support, and $\mathbf{P}$ is improper. Note that $(0,0) \notin S(\mathbf{P}), g_{1}=1$, and $e_{1}=0$. Let $s^{\prime}=s, t^{\prime}=t / s$ be the new parameters. We obtain a reparametrize:

$$
\mathbf{P}^{\prime}\left(s^{\prime}, t^{\prime}\right)=\left(1+s^{\prime 2}+\left(1+s^{\prime 2}\right) t^{\prime}, 1+2 s^{\prime 2}+\left(1-s^{\prime 2}\right) t^{\prime}, s^{\prime 2}+t^{\prime}, 1+t^{\prime}\right) .
$$

The support for $\mathbf{P}^{\prime}$ is $S\left(\mathbf{P}^{\prime}\right)=\{(0,0),(2,0),(0,1),(2,1)\}, \operatorname{gcddg}\left(\mathbf{P}^{\prime}\right)=2$ and $(0,0) \in S\left(\mathbf{P}^{\prime}\right)$. Using $\bar{s}=s^{\prime 2}, \bar{t}=t^{\prime}$ to reparametrize $\mathbf{P}^{\prime}$, we obtain a new parametrization of $\mathbf{P}$ :

$$
\overline{\mathbf{P}}(\bar{s}, \bar{t})=(1+\bar{s}+(1+\bar{s}) \bar{t}, 1+2 \bar{s}+(1-\bar{s}) \bar{t}, \bar{s}+\bar{t}, 1+\bar{t}) .
$$

We have $S(\overline{\mathbf{P}})=\{(0,0),(1,0),(0,1),(1,1)\}$ and $\operatorname{gcddg}(\overline{\mathbf{P}})=1$.

The above result shows that if $\operatorname{gcddg}(\mathbf{P})>1$, we may reparametrize the parametrization such that the degree gap of the new parametrization is one.

In the rest of this paper, we will give a proper reparametrization algorithm for those improper parametrizations with $\operatorname{gcddg}(\mathbf{P})=1$. 


\section{The Proper Parametrization Algorithm}

\subsection{Proper Reparametrization of Rational Curves}

Our algorithm needs a proper reparametrization algorithm for algebraic curves. There exist several such algorithms. The method given in [10] is the simplest one and will be used in this paper.

Theorem 3.1 [10] Let

$$
\mathbf{C}(s)=\left(\frac{P_{11}(s)}{P_{12}(s)}, \frac{P_{21}(s)}{P_{22}(s)}\right)
$$

be a rational planar curve, where $P_{i j}(s) \in \mathbb{Q}[s], i, j=1,2$. Let

$$
\begin{aligned}
& H_{1}(s, \bar{s})=P_{11}(s) P_{12}(\bar{s})-P_{12}(s) P_{11}(\bar{s}), \\
& H_{2}(s, \bar{s})=P_{21}(s) P_{22}(\bar{s})-P_{22}(s) P_{21}(\bar{s}), \\
& H(s, \bar{s})=\operatorname{gcd}\left(H_{1}, H_{2}\right) .
\end{aligned}
$$

If $H=c(s-\bar{s})$ for $c \in \mathbb{Q}$, then $\mathbf{C}(s)$ is proper. Otherwise, write $H$ as a polynomial in $\bar{s}$ :

$$
H=c_{d} \bar{s}^{d}+\cdots+c_{1} \bar{s}+c_{0}, c_{d} \neq 0, c_{i} \in \mathbb{Q}[s], i=0, \ldots, d .
$$

Then, there exist $k, l, k \neq l$ such that $\frac{c_{k}}{c_{l}} \notin \mathbb{Q}$ and $\bar{s}=\bar{s}(s)=\frac{c_{k}}{c_{l}}$ is a new parameter for the curve.

Furthermore, let $L_{i}\left(\bar{s}, x_{i}\right)=\operatorname{resl}\left(x_{i} P_{i 2}(s)-P_{i 1}(s), c_{l} \bar{s}-c_{k}, s\right), i=1,2$ be the resultant wrt s. Then, $L_{i}\left(\bar{s}, x_{i}\right)=\left(Q_{i 2} x_{i}-Q_{i 1}\right)^{\operatorname{deg}(\bar{s}, s)}, i=1,2$, and

$$
\overline{\mathbf{C}}(\bar{s})=\left(\frac{Q_{11}(\bar{s})}{Q_{12}(\bar{s})}, \frac{Q_{21}(\bar{s})}{Q_{22}(\bar{s})}\right)
$$

is a proper reparametrization of the curve $\mathbf{C}(s)$.

\subsection{Proper Reparametrization for Parameter $t$}

Example 2.1 shows that an improper parametrization of form (1) for a ruled surface is generally not proper for the variable $t$. In this section, we will first reparametrize such an improper parametrization to make the new parametrization proper for the variable $t$ in affine form.

Theorem 3.2 Let $\mathbf{P}(s, t)$ be a rational ruled surface of form (1). Then we can reparameterize $\mathbf{P}(s, t)$ as $\overline{\mathbf{P}}(\bar{s}, \bar{t})$ such that $\bar{t}$ is proper in $\overline{\mathbf{P}}(\bar{s}, \bar{t})$. More precisely, we have

$$
\overline{\mathbf{P}}(\bar{s}, \bar{t})=\left(\bar{a}_{0}(\bar{s})+\bar{a}_{1}(\bar{s}) \bar{t}, \bar{b}_{0}(\bar{s})+\bar{b}_{1}(\bar{s}) \bar{t}, \bar{d}(\bar{s}) \bar{t}, \bar{d}(\bar{s})\right),
$$

where $\bar{a}_{i}, \bar{b}_{i}, \bar{d} \in \mathbb{Q}[\bar{s}], i=0,1$.

Proof: $\mathbf{P}(s, t)=\left(a_{0}(s)+a_{1}(s) t, b_{0}(s)+b_{1}(s) t, c_{0}(s)+c_{1}(s) t, d_{0}(s)+d_{1}(s) t\right)$ is a rational ruled surface, $d_{0}, d_{1}$ can not be zero simultaneously.

If $d_{1}=0$, not all $a_{1}(s), b_{1}(s), c_{1}(s)$ is zero since $\mathbf{P}$ is a rational ruled surface. Without loss of generality, we assume $c_{1}(s) \neq 0$. Let

$$
\bar{t}=\frac{c_{0}(s)+c_{1}(s) t}{d_{0}(s)}, \bar{s}=s
$$


be the new parameters. Substituting $t=\frac{d_{0}(\bar{s}) \bar{t}-c_{0}(\bar{s})}{c_{1}(\bar{s})}, s=\bar{s}$ into (1), we have

$$
\begin{aligned}
\overline{\mathbf{P}}(\bar{s}, \bar{t}) & \\
= & \left(\frac{a_{0}(\bar{s}) c_{1}(\bar{s})-a_{1}(\bar{s}) c_{0}(\bar{s})+a_{1}(\bar{s}) d_{0}(\bar{s}) \bar{t}}{c_{1}(\bar{s}) d_{0}(\bar{s})}, \frac{b_{0}(\bar{s}) c_{1}(\bar{s})-b_{1}(\bar{s}) c_{0}(\bar{s})+b_{1}(\bar{s}) d_{0}(\bar{s}) \bar{t}}{c_{1}(\bar{s}) d_{0}(\bar{s})}, \bar{t}, 1\right) \\
= & \left(a_{0}(\bar{s}) c_{1}(\bar{s})-a_{1}(\bar{s}) c_{0}(\bar{s})+a_{1}(\bar{s}) d_{0}(\bar{s}) \bar{t}, b_{0}(\bar{s}) c_{1}(\bar{s})-b_{1}(\bar{s}) c_{0}(\bar{s})+b_{1}(\bar{s}) d_{0}(\bar{s}) \bar{t},\right. \\
& \left.c_{1}(\bar{s}) d_{0}(\bar{s}) \bar{t}, c_{1}(\bar{s}) d_{0}(\bar{s})\right) .
\end{aligned}
$$

If $d_{1} \neq 0$, let

$$
t^{\prime}=\frac{1}{d_{0}(s)+d_{1}(s) t}, s=s^{\prime}
$$

be the new parameters. Substituting $s=s^{\prime}, t=\frac{1-d_{0}\left(s^{\prime}\right) t^{\prime}}{d_{1}\left(s^{\prime}\right) t^{\prime}}$ into $\mathbf{P}(s, t)$, we have

$$
\begin{aligned}
\mathbf{P}^{\prime}\left(s^{\prime}, t^{\prime}\right) & \left(\frac{\left(a_{0}\left(s^{\prime}\right) d_{1}\left(s^{\prime}\right)-a_{1}\left(s^{\prime}\right) d_{0}\left(s^{\prime}\right)\right) t^{\prime}+a_{1}\left(s^{\prime}\right)}{d_{1}\left(s^{\prime}\right)}, \frac{\left(b_{0}\left(s^{\prime}\right) d_{1}\left(s^{\prime}\right)-b_{1}\left(s^{\prime}\right) d_{0}\left(s^{\prime}\right)\right) t^{\prime}+b_{1}\left(s^{\prime}\right)}{d_{1}\left(s^{\prime}\right)}\right. \\
& \left.\frac{\left(c_{0}\left(s^{\prime}\right) d_{1}\left(s^{\prime}\right)-c_{1}\left(s^{\prime}\right) d_{0}\left(s^{\prime}\right)\right) t^{\prime}+c_{1}\left(s^{\prime}\right)}{d_{1}\left(s^{\prime}\right)}, 1\right) \\
= & \left(\left(a_{0}\left(s^{\prime}\right) d_{1}\left(s^{\prime}\right)-a_{1}\left(s^{\prime}\right) d_{0}\left(s^{\prime}\right)\right) t^{\prime}+a_{1}\left(s^{\prime}\right),\left(b_{0}\left(s^{\prime}\right) d_{1}\left(s^{\prime}\right)-b_{1}\left(s^{\prime}\right) d_{0}\left(s^{\prime}\right)\right) t^{\prime}+b_{1}\left(s^{\prime}\right)\right. \\
& \left.\left(c_{0}\left(s^{\prime}\right) d_{1}\left(s^{\prime}\right)-c_{1}\left(s^{\prime}\right) d_{0}\left(s^{\prime}\right)\right) t^{\prime}+c_{1}\left(s^{\prime}\right), d_{1}\left(s^{\prime}\right)\right)
\end{aligned}
$$

which becomes case one. This proves the theorem

Corollary 3.3 $\mathbf{P}(s, t)$ is proper if and only if $\overline{\mathbf{P}}(\bar{s}, \bar{t})$ is proper.

Proof: Note that the transformations (5) and (6) are birational transformations of the same rational ruled surface. Hence we have the result.

Example 3.4 Continuing from Example 2.1, for the improper parametrization

$$
\mathbf{P}(s, t)=(3 s+(s+1) t, 2 s+s t, s-1+t, 1)
$$

of the plane $x-y-z-w=0$, let $\bar{t}=s-1+t, \bar{s}=s$. Substituting $t=\bar{t}-\bar{s}+1, s=\bar{s}$ into $\mathbf{P}$, we have the new parametrization of $\mathbf{P}(s, t)$

$$
\overline{\mathbf{P}}(\bar{s}, \bar{t})=\left(1+3 \bar{s}-\bar{s}^{2}+(\bar{s}+1) \bar{t}, 3 \bar{s}-\bar{s}^{2}+\bar{s} \bar{t}, \bar{t}, 1\right)
$$

which is proper for $\bar{t}$.

\subsection{Proper Reparametrization of Ruled Surfaces}

From Theorem 3.2, we may assume that the ruled surface has the following form in affine space:

$$
\left\{\begin{array}{l}
x=\frac{a_{0}(s)+a_{1}(s) t}{d(s)} \\
y=\frac{b_{0}(s)+b_{1}(s) t}{d(s)} \\
z=t
\end{array}\right.
$$


where $a_{0}, a_{1}, b_{0}, b_{1}, d \in \mathbb{Q}[s]$.

Our idea is to treat (7) as a rational parametrization of a planar algebraic curve of $(x, y)$ with parameter $s$ and with coefficients in $\mathbb{Q}(t)$. Then we can find a proper parametrization for this curve and show that this reparametrization also provides a proper reparametrization for the ruled surface.

As a consequence of Theorem 3.1, we have the following result.

Theorem 3.5 Consider a ruled surface (7). Let

$$
\begin{aligned}
& H_{1}(s, \bar{s})=d(s)\left(a_{0}(\bar{s})+a_{1}(\bar{s}) t\right)-\left(a_{0}(s)+a_{1}(s) t\right) d(\bar{s}), \\
& H_{2}(s, \bar{s})=d(s)\left(b_{0}(\bar{s})+b_{1}(\bar{s}) t\right)-\left(b_{0}(s)+b_{1}(s) t\right) d(\bar{s}), \\
& H(s, \bar{s})=\operatorname{gcd}\left(H_{1}, H_{2}\right) .
\end{aligned}
$$

If $H=c(s-\bar{s})$ for $c \in \mathbb{Q}[t]$, then (7) is proper; otherwise, write $H$ as a polynomial in $\bar{s}$ :

$$
H=c_{d} \bar{s}^{d}+\cdots+c_{1} \bar{s}+c_{0}, c_{d} \neq 0, c_{i} \in \mathbb{Q}[t][s], i=0, \ldots, d .
$$

Then there exist $k, l, k \neq l$ such that $\frac{c_{k}}{c_{l}} \notin \mathbb{Q}(t)$, and a set of new parameters for the surface are

$$
\bar{s}=\frac{c_{k}(s, t)}{c_{l}(s, t)}, \bar{t}=t
$$

Furthermore, let

$$
L_{1}(\bar{s}, x)=\operatorname{resl}\left(G_{1}(s, x), c_{l} \bar{s}-c_{k}, s\right), L_{2}(\bar{s}, y)=\operatorname{resl}\left(G_{2}(s, y), c_{l} \bar{s}-c_{k}, s\right)
$$

where $G_{1}(s, x)=x d(s)-\left(a_{0}(s)+a_{1}(s) t\right), G_{2}(s, y)=y d(s)-\left(b_{0}(s)+b_{1}(s) t\right)$. Then

$$
L_{1}=\left(Q_{12}(\bar{s}, t) x-Q_{11}(\bar{s}, t)\right)^{\operatorname{deg}(\bar{s}, s)}, L_{2}=\left(Q_{22}(\bar{s}, t) y-Q_{21}(\bar{s}, t)\right)^{\operatorname{deg}(\bar{s}, s)}
$$

where $Q_{i j} \in \mathbb{Q}[\bar{s}, t]$. An proper reparametrization of (7) using the new parameters $\bar{s}, \bar{t}$ is $\left(\frac{Q_{11}(\bar{s}, \bar{t})}{Q_{12}(\bar{s}, \bar{t})}, \frac{Q_{21}(\bar{s}, \bar{t})}{Q_{22}(\bar{s}, \bar{t})}, \bar{t}\right)$.

Proof: We can consider

$$
\left\{\begin{array}{l}
x=\frac{a_{0}(s)+a_{1}(s) t}{d(s)} \\
y=\frac{b_{0}(s)+b_{1}(s) t}{d(s)}
\end{array}\right.
$$

as a planar curve with base field $\mathbb{Q}(t)$. According to Theorem 3.1,

$$
\left\{\begin{array}{l}
x=\frac{Q_{11}(\bar{s}, \bar{t})}{Q_{12}(\bar{s}, \bar{t})} \\
y=\frac{Q_{21}(\bar{s}, \bar{t})}{Q_{22}(\bar{s}, \bar{t})}
\end{array}\right.
$$

is a proper reparametrization of (8). Let the inversion map of (9) be $\bar{s}=r(x, y, \bar{t})$, where $r(x, y, \bar{t}) \in \mathbb{Q}(x, y, \bar{t})$. Since $z=\bar{t}=t$. The inversion, map for $(7)$ is $\bar{s}=r(x, y, z), \bar{t}=z$. Therefore,

$$
\left(\frac{Q_{11}(\bar{s}, \bar{t})}{Q_{12}(\bar{s}, \bar{t})}, \frac{Q_{21}(\bar{s}, \bar{t})}{Q_{22}(\bar{s}, \bar{t})}, \bar{t}\right)
$$

is a proper reparametrization of (7). 
Example 3.6 In Example 3.4, we obtain the following new parametrization of the ruled surface

$$
\overline{\mathbf{P}}(\bar{s}, \bar{t})=\left(1+3 \bar{s}-\bar{s}^{2}+(\bar{s}+1) \bar{t}, 3 \bar{s}-\bar{s}^{2}+\bar{s} \bar{t}, \bar{t}, 1\right)
$$

which is proper for $\bar{t}$, but not proper for $\bar{s}$.

Following Theorem 3.5, the corresponding affine form of $\overline{\mathbf{P}}(\bar{s}, \bar{t})$ is

$$
(x, y, z)=\overline{\mathbf{A P}}=\left(1+3 \bar{s}-\bar{s}^{2}+(\bar{s}+1) \bar{t}, 3 \bar{s}-\bar{s}^{2}+\bar{s} \bar{t}, \bar{t}\right) .
$$

We treat the first two parameters of $\overline{\mathbf{A P}}$ as a rational parametrization of an algebraic curve with parameter $s$ with coefficients in the field $\mathbb{Q}(\bar{t})$.

Using Theorem 3.1, we have

$$
\begin{gathered}
H_{1}\left(\bar{s}, s^{\prime}\right)=\left(1+3 s^{\prime}-s^{\prime 2}+\left(s^{\prime}+1\right) \bar{t}\right)-\left(1+3 \bar{s}-\bar{s}^{2}+(\bar{s}+1) \bar{t}\right), \\
H_{2}\left(\bar{s}, s^{\prime}\right)=\left(3 s^{\prime}-s^{\prime 2}+s^{\prime} \bar{t}\right)-\left(3 \bar{s}-\bar{s}^{2}+\bar{s} \bar{t}\right), \\
H=s^{\prime 2}+(-\bar{t}-3) s^{\prime}-\bar{s}^{2}+\bar{s} \bar{t}+3 \bar{s} .
\end{gathered}
$$

Then $c_{2}=1, c_{1}=(-\bar{t}-3), c_{0}=-\bar{s}^{2}+\bar{s} \bar{t}+3 \bar{s}$. We obtain the new parameters $s^{\prime}=c_{0} / c_{2}=$ $3 \bar{s}-\bar{s}^{2}+\bar{t} \bar{s}, t^{\prime}=\bar{t}$ and a proper reparametrization of $\overline{\mathbf{A P}}$

$$
\left(s^{\prime}+1+t^{\prime}, s^{\prime}, t^{\prime}\right) \text {. }
$$

Corollary 3.7 In Theorem 3.5, if $c_{k}, c_{l} \in \mathbb{Q}[s]$, then the parameters $s$ and $t$ can be separated. More precisely, we can obtain a new parameter $\bar{s}=\frac{\psi_{1}(s)}{\psi_{2}(s)}$ free of $t$. The new parametrization (10) is linear in $\bar{t}$ and $Q_{12}$ and $Q_{22}$ are free of $\bar{t}$.

Proof: If $c_{k}, c_{l} \in \mathbb{Q}[s]$, then the new parameter is $\bar{s}=\frac{c_{k}}{c_{l}} \in \mathbb{Q}(s)$. So the parameters $s$ and $t$ can be separated. We will prove that the new parametrization is also linear in $\bar{t}$. We obtain the new parametrization by computing resultants. Note that $G_{1}$ is linear in $t$ and $x$, and $c_{k} \bar{s}-c_{l}$ does not contain the parameter $t$. So $Q_{12}(\bar{s}) x-Q_{11}(\bar{s})$ has the same degree in $x$ and $t$, and hence is linear in $t$. More precisely, the coefficient of $x$ in $G_{1}$ is free of $t$, so the leading coefficient of the resultant is free of $t$. It means that $Q_{12}$ is free of $t$. The same is true for $Q_{22}(\bar{s}) y-Q_{21}(\bar{s})$. We prove the corollary.

In Theorem 3.5, we reparametrize the ruled surface in the affine space. The new parametric ruled surface is still proper when we consider it in homogenous form.

Theorem 3.8 Let $\mathbf{P}(s, t)=(a(s, t), b(s, t), c(s, t), d(s, t))$ as (1) be the rational ruled surface in homogenous form, and

$$
\mathbf{A P}(s, t)=\left(\frac{a(s, t)}{d(s, t)}, \frac{b(s, t)}{d(s, t)}, \frac{c(s, t)}{d(s, t)}\right)
$$

a parametric surface of (1) in affine space. Then $\mathbf{P}$ is a proper parametrization in homogenous form if and only if $\mathbf{A P}$ is a proper parametrization in affine space. 
Proof: Assume the algebraic degree of the ruled surface to be $n$ which is the degree of the implicit equation of the ruled surface and the improper index of (1) and (11) to be $I X_{1}$ and $I X_{2}$ respectively. To prove this theorem, we need only to prove $I X_{1}=I X_{2}$. According to [7], we can compute the improper index of a surface by computing the number of intersections of a generic line with this surface. We use two generic planes to represent a generic line:

$$
\left\{\begin{array}{l}
u_{1} x+u_{2} y+u_{3} z+u_{0} w=0 \\
v_{1} x+v_{2} y+v_{3} z+v_{0} w=0
\end{array}\right.
$$

In projective space the number of solutions of

$$
\left\{\begin{array}{l}
u_{1} a(s, t)+u_{2} b(s, t)+u_{3} c(s, t)+u_{0} d(s, t)=0 \\
v_{1} a(s, t)+v_{2} b(s, t)+v_{3} c(s, t)+v_{0} d(s, t)=0
\end{array}\right.
$$

is $n I X_{1}+\alpha$, where $\alpha$ is the number of the common zero of $a(s, t), b(s, t), c(s, t), d(s, t)[3]$.

In affine space, we consider the solutions of the polynomial equations obtained by substituting (11) into (12) with $w=1$,

$$
\left\{\begin{array}{l}
u_{1} \frac{a(s, t)}{d(s, t)}+u_{2} \frac{b(s, t)}{d(s, t)}+u_{3} \frac{c(s, t)}{d(s, t)}+u_{0}=0, \\
v_{1} \frac{a(s, t)}{d(s, t)}+v_{2} \frac{b(s, t)}{d(s, t)}+v_{3} \frac{c(s, t)}{d(s, t)}+v_{0}=0 .
\end{array}\right.
$$

The number of the solutions of (14) is $n I X_{2}$. And the solutions of (14) are the solutions of (13) by removing solutions of

$$
\left\{\begin{array}{l}
u_{1} a(s, t)+u_{2} b(s, t)+u_{3} c(s, t)+u_{0} d(s, t)=0 \\
v_{1} a(s, t)+v_{2} b(s, t)+v_{3} c(s, t)+v_{0} d(s, t)=0 \\
d(s, t)=0
\end{array}\right.
$$

Now, we prove that (15) has $\alpha$ solutions. Computing the resultant of the first two equations of (15) with respect to $t$. Note that these two equations are both linear in $t$, we have:

$$
R e s l=c(u, v) \prod_{i=1}^{\alpha}\left(s-\alpha_{i}\right) \prod_{j}\left(s-s_{j}\right),
$$

where $c(u, v) \in \mathbb{Q}[u, v]$ and $\alpha_{i}$ are the $s$-coordinates corresponding to the common zeros of $a(s, t), b(s, t), c(s, t), d(s, t)$ and $s_{j}$ are the $s$-coordinates corresponding to other solutions of (13).

If (15) has more than $\alpha$ solutions, then there is at least one $j$, say $l$, such that $d\left(s_{l}(u, v), t_{l}(u, v)\right) \equiv$ 0 . Because $s_{l}$ is not a constant, at least one $u_{k}$ or $v_{k}$, say $u_{0}$, occurs in its expression. Note that Resl is symmetrical for $u_{0}$ and $v_{0}$, so $v_{0}$ also occurs in $s_{l}$. Therefore, $s_{l}$ contains at least two free parameters. On the other hand, $d(s, t)=0$ is a curve and $d\left(s_{l}(u, v), t_{l}(u, v)\right) \not \equiv 0$, a contradiction. So $n I X_{1}=n I X_{2}$, and hence $I X_{1}=I X_{2}$.

\subsection{The Algorithm}

Now we can give the proper reparametrization algorithm. 


\section{Algorithm 3.9 The Proper Reparametrization Algorithm}

Input: $\mathbf{P}(s, t)=\left(a_{0}(s)+a_{1}(s) t, b_{0}(s)+b_{1}(s) t, c_{0}(s)+c_{1}(s) t, d_{0}(s)+d_{1}(s) t\right)$.

Output: If $\mathbf{P}$ is proper, then return "P is proper." Otherwise, return a proper reparametrization of $\mathbf{P}(s, t)$.

S1 If $d_{1}=0$, goto $\mathbf{S 2}$.

Let $t^{\prime}=\frac{1}{d_{0}(s)+d_{1}(s) t}$ and substituting $t=\frac{1-d_{0}(s) t^{\prime}}{d_{1}(s) t^{\prime}}$ into $\mathbf{P}(s, t)$. We obtain a new reparametrization $\mathbf{P}^{\prime}\left(s, t^{\prime}\right)$.

Let $\mathbf{P}(s, t)=\mathbf{P}^{\prime}(s, t)$.

S2 Without loss of generality, we may assume $c_{1}(s) \neq 0$. Let $\bar{t}=\frac{c_{0}(s)+c_{1}(s) t}{d(s)}$ and substituting $t=\frac{d(s) \bar{t}-c_{0}(s)}{c_{1}(s)}$ into $\mathbf{P}$. We obtain a new reparametrization:

$$
\left\{\begin{array}{l}
x=\frac{\bar{a}_{0}(s)+\bar{a}_{1}(s) \bar{t}}{\bar{d}(s)} \\
y=\frac{\bar{b}_{0}(s)+\bar{b}_{1}(s) \bar{t}}{\bar{d}(s)} \\
z=\bar{t}
\end{array}\right.
$$

S3 Compute

$$
\begin{aligned}
& H_{1}(s, \bar{s})=\bar{d}(s)\left(\bar{a}_{0}(\bar{s})+\bar{a}_{1}(\bar{s}) \bar{t}\right)-\left(\bar{a}_{0}(s)+\bar{a}_{1}(s) \bar{t}\right) \bar{d}(\bar{s}), \\
& H_{2}(s, \bar{s})=\bar{d}(s)\left(\bar{b}_{0}(\bar{s})+\bar{b}_{1}(\bar{s}) \bar{t}\right)-\left(\bar{b}_{0}(s)+\bar{b}_{1}(s) \bar{t}\right) \bar{d}(\bar{s}), \\
& H(s, \bar{s})=\operatorname{gcd}\left(H_{1}, H_{2}\right) .
\end{aligned}
$$

If $H=c(s-\bar{s})$ for $c \in \mathbb{Q}[\bar{t}]$, return: " $P(s)$ is proper."

S4 Write $S$ as a polynomial in $\bar{s}$ :

$$
H=c_{d} \bar{s}^{d}+\cdots+c_{1} \bar{s}+c_{0}, c_{d} \neq 0, c_{i} \in Q[\bar{t}][s], i=0, \ldots, d,
$$

There exists a $k \neq d$ such that $\frac{c_{k}}{c_{d}} \notin Q(t)$.

S5 Let $D=\max \left\{\operatorname{deg}\left(c_{d}, s\right), \operatorname{deg}\left(c_{k}, s\right)\right\}$. Compute

$$
\begin{aligned}
& L_{1}(\bar{s}, x)=\operatorname{resl}\left(G_{1}(s, x), c_{d} \bar{s}-c_{k}, s\right)=\left(Q_{12}(\bar{s}) x-Q_{11}(\bar{s})\right)^{D}, \\
& L_{2}(\bar{s}, y)=\operatorname{resl}\left(G_{2}(s, y), c_{d} \bar{s}-c_{k}, s\right)=\left(Q_{22}(\bar{s}) y-Q_{21}(\bar{s})\right)^{D},
\end{aligned}
$$

where $G_{1}(s, x)=x d(s)-\left(a_{0}(s)+a_{1}(s) \bar{t}\right), G_{2}(s, y)=y d(s)-\left(b_{0}(s)+b_{1}(s) \bar{t}\right)$.

S6 Let

$$
\begin{gathered}
\left(Q_{1}(\bar{s}, \bar{t}), Q_{2}(\bar{s}, \bar{t}), Q_{3}(\bar{s}, \bar{t}), Q_{4}(\bar{s}, \bar{t})\right)=\left(Q_{11} Q_{22}, Q_{21} Q_{12}, Q_{12} Q_{22} \bar{t}, Q_{12} Q_{22}\right), \\
F=\operatorname{gcd}\left(Q_{1}, Q_{2}, Q_{3}, Q_{4}\right) .
\end{gathered}
$$

Return

$$
\left(\frac{Q_{1}(\bar{s}, \bar{t})}{F}, \frac{Q_{2}(\bar{s}, \bar{t})}{F}, \frac{Q_{3}(\bar{s}, \bar{t})}{F}, \frac{Q_{4}(\bar{s}, \bar{t})}{F}\right) .
$$

Remark 3.10 It is easy to see that the algorithm works for rational parametrizations of the affine form $\mathbf{A P}(s, t)=\left(r_{1}(s, t), r_{2}(s, t), r_{3}(s, t)\right)$ where $r_{i} \in \mathbb{Q}(s, t)$ and one of $r_{i}$ is linear in $s$ or $t$. 


\section{Conclusions}

In this paper, we give a proper reparametrization algorithm for rational ruled surfaces. In general, we cannot guarantee the new parametrization is still linear in one of the variables. It is an interesting problem to find a proper reparametrization for a rational ruled surface which is still of form (1).

\section{References}

[1] F.L. Chen, J.M. Zheng and T. W. Sederberg, The $\mu$-basis of a Rational Ruled Surface, Computer Aided Geometric Design, 18, 61-72, 2001.

[2] F.L. Chen and W.P. Wang, Revisiting the $\mu$-basis of a Rational Ruled Surface, Journal of Symbolic Computation, 36, 699-716, 2003.

[3] E.W. Chionh and R.N. Goldman, Degree, Multiplicity, and Inversion Formulas for Rational Surfaces using U-resultants, Computer Aided Geometric Design, 9, 93-108, 1992.

[4] E.W. Chionh, X.S. Gao, and L.Y. Shen, Inherently Improper Surface Parametric Supports, Computer Aided Geometric Design, 23, 629-639, 2006.

[5] X.S. Gao and S.C. Chou, Implicitization of Rational Parametric Equations, Journal of the Symbolic Computation, 14, 459-470, 1992.

[6] A. Schinzel, Polynomials wih Special Regard to Reducibility, Cambridge University Press, 2000.

[7] B.L. Van der Waerden, Einfürung in Die Algebraischen Geometrie, Springer Verlag, Berlin, 1973.

[8] J. Li and X.S. Gao, The Proper Parametrization of a Special Class of Rational Parametric Equations, Journal of Systems Science and Complexity,19, 331-339, 2006.

[9] T.W. Sederberg, Improperly Parametrized Rational Curves, Computer Aided Geomeric Design, 3, 67-75, 1986.

[10] P.D. Sonia, On the Problem of Proper Reparametrization for Rational Curves and Surfaces, Computer Aided Geometric Design, 23, 307-323, 2006.

[11] R.J. Walker, Algebraic Curves, Princeton University Press, 1950. 